SSU-HEP-16/07

\title{
Vacuum polarization and quadrupole corrections to the hyperfine splitting of P-states in muonic deuterium
}

\author{
A. P. Martynenko and V. V. Sorokin \\ Samara University, Moskovskoye Shosse 34, 443086, Samara, Russia
}

\begin{abstract}
On the basis of quasipotential approach in quantum electrodynamics we calculate vacuum polarization and quadrupole corrections in first and second orders of perturbation theory in hyperfine structure of P-states in muonic deuterium. All corrections are presented in integral form and evaluated analytically and numerically. The obtained results can be used for the improvement of the transition frequencies between levels $2 \mathrm{P}$ and $2 \mathrm{~S}$.
\end{abstract}

PACS numbers: 31.30.jf, 12.20.Ds, 36.10.Ee

Keywords: Hyperfine structure, muonic atoms, quantum electrodynamics.

In last years a significant theoretical interest in the investigation of fine and hyperfine energy structure of simple atoms is related with light muonic atoms: muonic hydrogen, muonic deuterium and ions of muonic helium. This is conditioned due to essential progress achieved by experimental collaboration CREMA (Charge Radius Experiment with Muonic Atoms) in studies of such simple atoms [1, 2]. In these experiments, the aim is to clarify the values of the charge radii of nuclei. The measurement of the transition frequency $\left(2 S_{1 / 2}^{f=1}-2 P_{3 / 2}^{f=2}\right)$ leads to a new more precise value of the proton charge radius. For the first time the hyperfine splitting (HFS) of $2 \mathrm{~S}$ state in muonic hydrogen was measured in [2]. Analogous measurements in muonic deuterium and muonic helium ions are also carried out [3]. The experiments with muonic hydrogen and deuterium have shown that there are significant discrepancies between the values of charge radii of the proton and deuteron obtained from experiments with electronic and muonic atoms. Successful realization of experimental program is based on precise theoretical calculations of different corrections to the energy intervals of fine and hyperfine structure of muonic atoms [4 -16]. The magnitude of various theoretical contributions to the energy levels is determined by the values of fundamental physical constants. The emerging discrepancy requires new analysis of various corrections in the spectrum, in spite of the fact that the initial estimate of some of them has a small value. Contributions connected with the structure of nuclei and vacuum polarization as well as combined corrections have in the calculations the increasing importance. In this work we investigate a special class of corrections on deuteron structure and vacuum polarization for hyperfine splitting of P-states in muonic deuterium. In contrast to our previous work [17], we use the coordinate representation to calculate the various matrix elements.

Let us consider the HFS of P-states in muonic deuterium. Our approach is based on quasipotential method in quantum electrodynamics (QED) [18 20], in which two-particle bound state is described by the Schrödinger equation. Main contribution to hyperfine split- 
ting in muonic deuterium is given by hyperfine part of the Breit Hamiltoian [17, 21, 22]:

$\Delta V_{B}^{h f s}(r)=\frac{Z \alpha\left(1+\kappa_{d}\right)}{2 m_{1} m_{2} r^{3}}\left[1+\frac{m_{1} \kappa_{d}}{m_{2}\left(1+\kappa_{d}\right)}\right]\left(\boldsymbol{L} \cdot \boldsymbol{s}_{2}\right)-\frac{Z \alpha\left(1+\kappa_{d}\right)\left(1+a_{\mu}\right)}{2 m_{1} m_{2} r^{3}}\left[\left(\boldsymbol{s}_{1} \cdot \boldsymbol{s}_{2}\right)-3\left(\boldsymbol{s}_{1} \cdot \boldsymbol{n}\right)\left(\boldsymbol{s}_{2} \cdot \boldsymbol{n}\right)\right]$,

where $m_{1}, m_{2}$ are muon and deuteron masses respectively, $\kappa_{d}, a_{\mu}$ are anomalous magnetic moments of deuteron and muon, $\boldsymbol{L}, \boldsymbol{s}_{1}$ are orbital momentum and spin of muon, $\boldsymbol{s}_{2}=\mathbf{I}$ is the deuteron spin, $\boldsymbol{n}=\boldsymbol{r} / r$. This operator doesn't commute with the operator of total angular momentum of muon $\boldsymbol{J}=\boldsymbol{L}+\boldsymbol{s}_{1}$, which leads to non-zero off-diagonal matrix elements. The Coulomb wave function for $2 \mathrm{P}$-state has the following form:

$$
\Psi_{2 P}(\boldsymbol{r})=\frac{1}{2 \sqrt{6}} W^{\frac{5}{2}} r e^{-\frac{W r}{2}} Y_{1 m}(\theta, \phi), \quad W=\mu Z \alpha .
$$

Averaging (11) over wave function (2) we obtain the contributions of order $\alpha^{4}$ to HFS of P-states which are written analytically in [17] and numerically in Table I. The one-loop vacuum polarization correction to the operator (11) has the following form [23]:

$$
\begin{aligned}
& \Delta V_{1 \gamma, v p}^{h f s}(r)=\frac{Z \alpha^{2}\left(1+\kappa_{d}\right)}{6 \pi m_{1} m_{2} r^{3}} \int_{1}^{\infty} \rho(\xi) d \xi e^{-2 m_{e} \xi r}\left\{\left(1+\frac{m_{1} \kappa_{d}}{m_{2}\left(1+\kappa_{d}\right)}\right)\left(\boldsymbol{L} \cdot \boldsymbol{s}_{2}\right)\left(1+2 m_{e} \xi r\right)-(3)\right. \\
& \left.\left(1+a_{\mu}\right)\left(4 m_{e}^{2} \xi^{2} r^{2}\left[\left(\boldsymbol{s}_{1} \cdot \boldsymbol{s}_{2}\right)-\left(\boldsymbol{s}_{1} \cdot \boldsymbol{n}\right)\left(\boldsymbol{s}_{2} \cdot \boldsymbol{n}\right)\right]+\left(1+2 m_{e} \xi r\right)\left[\left(\boldsymbol{s}_{1} \cdot \boldsymbol{s}_{2}\right)-3\left(\boldsymbol{s}_{1} \cdot \boldsymbol{n}\right)\left(\boldsymbol{s}_{2} \cdot \boldsymbol{n}\right)\right]\right)\right\} .
\end{aligned}
$$

The contribution of (3) to hyperfine splitting is given by integral expression:

$$
\begin{aligned}
E_{1 \gamma, v p}^{h f s}(r)= & \frac{\alpha^{4} \mu^{3}\left(1+\kappa_{d}\right)}{24 m_{1} m_{2} r^{3}} \frac{\alpha}{6 \pi} \int_{1}^{\infty} \rho(\xi) d \xi \int_{0}^{\infty} x d x e^{-x\left[1+\frac{2 m_{e} \xi}{W}\right]}\left[\left(1+\frac{m_{1} \kappa_{d}}{m_{2}\left(1+\kappa_{d}\right)}\right) \times\right. \\
& \left.\overline{T_{1}}\left(1+\frac{2 m_{e} \xi}{W} x\right)-\left(1+a_{\mu}\right)\left(\frac{4 m_{e}^{2} \xi^{2} x^{2}}{W^{2}} \overline{T_{3}}+\left(1+\frac{2 m_{e} \xi}{W} x\right) \overline{T_{2}}\right)\right],
\end{aligned}
$$

where we introduce the following designations for the operators $T_{i}(\mathrm{i}=1,2,3)$ :

$$
T_{1}=\left(\boldsymbol{L} \cdot \boldsymbol{s}_{2}\right), T_{2}=\left[\left(\boldsymbol{s}_{1} \cdot \boldsymbol{s}_{2}\right)-3\left(\boldsymbol{s}_{1} \cdot \boldsymbol{n}\right)\left(\boldsymbol{s}_{2} \cdot \boldsymbol{n}\right)\right], T_{3}=\left[\left(\boldsymbol{s}_{1} \cdot \boldsymbol{s}_{2}\right)-\left(\boldsymbol{s}_{1} \cdot \boldsymbol{n}\right)\left(\boldsymbol{s}_{2} \cdot \boldsymbol{n}\right)\right] .
$$

To calculate matrix elements $\overline{T_{i}}$ we use the atomic wave function

$$
\Psi_{F M}=\sum_{m} C(I j F ; M-m, m) \psi_{I M-m} \psi_{j m},
$$

where $M$ is the projection of total momentum $\mathbf{F}$ on the z-axis, $m$ is the projection of total muon momentum $\mathbf{j}$ on the z-axis. For the calculation diagonal and off-diagonal matrix elements we can use the Wigner-Eckart theorem which allows to express the initial matrix element of a scalar product of two rank 1 irreducible operators $T^{1}$ and $T^{2}$ through the reduced matrix elements [24, 25]:

$$
<j^{\prime} I F\left|\left(T^{1} \cdot T^{2}\right)\right| J I F>=(-1)^{I+j^{\prime}-F} W\left(j I j^{\prime} I ; F 1\right)<j^{\prime}|| T^{1}\left\|j><I|| T^{2}\right\| I>,
$$


where the Racah coefficients are connected with $6 \mathrm{~J}$-symbols by the following relation:

$$
W\left(j_{1} j_{2} j_{5} j_{4} ; j_{3} j_{4}\right)=(-1)^{-j_{1}-j_{2}-j_{4}-j_{5}}\left\{\begin{array}{lll}
j_{1} & j_{2} & j_{3} \\
j_{4} & j_{5} & j_{6}
\end{array}\right\} .
$$

So, for example, for the first matrix element $\bar{T}_{1}$ we have:

$$
\begin{gathered}
<j^{\prime} I F \mid\left(\mathbf{L s}_{2} \mid j I F>=(-1)^{j^{\prime}-j-F-I+l+3 / 3} \sqrt{(2 j+1)\left(2 j^{\prime}+1\right)}\left\{\begin{array}{lll}
j & I & F \\
I & j^{\prime} & 1
\end{array}\right\} \times\right. \\
\left\{\begin{array}{lll}
l & j^{\prime} & \frac{1}{2} \\
j & l & 1
\end{array}\right\}<l|| L|| l><I|| I|| I>=(-1)^{j^{\prime}-j-F-I+l+3 / 3} \sqrt{(2 j+1)\left(2 j^{\prime}+1\right)} \times \\
\sqrt{I(I+1)(2 I+1) l(l+1)(2 l+1)}\left\{\begin{array}{lll}
j & I & F \\
I & j^{\prime} & 1
\end{array}\right\}\left\{\begin{array}{lll}
l & j^{\prime} & \frac{1}{2} \\
j & l & 1
\end{array}\right\} .
\end{gathered}
$$

Calculating coefficients in right part of (9) we obtain numerical value $\bar{T}_{1}$ in the case of Pstates. Similar evaluation of other matrix elements $\bar{T}_{i}$ gives the following result for diagonal matrix elements with $j=j^{\prime}=1 / 2$ and $j=j^{\prime}=3 / 2$ and off-diagonal matrix elements with $j=1 / 2, j^{\prime}=3 / 2$ :

$$
\begin{aligned}
& \overline{T_{1}}\left(\frac{1}{2}, \frac{1}{2}\right)=-\overline{T_{2}}=-2 \overline{T_{3}}=-\frac{4}{3} \delta_{F \frac{1}{2}}+\frac{2}{3} \delta_{F \frac{3}{2}}, \overline{T_{1}}\left(\frac{3}{2}, \frac{3}{2}\right)=5 \overline{T_{2}}=\frac{5}{2} \overline{T_{3}}=-\frac{5}{3} \delta_{F \frac{1}{2}}-\frac{2}{3} \delta_{F \frac{3}{2}}+\delta_{F \frac{5}{2}}, \\
& \bar{T}_{1}\left(\frac{1}{2}, \frac{3}{2}\right)=2 \bar{T}_{2}\left(\frac{1}{2}, \frac{3}{2}\right)=-2 \bar{T}_{3}\left(\frac{1}{2}, \frac{3}{2}\right)=\left\{\begin{array}{l}
-\frac{\sqrt{2}}{3}, F=\frac{1}{2} \\
-\frac{\sqrt{5}}{3}, F=\frac{3}{2}
\end{array} .\right.
\end{aligned}
$$

The integration in (4) is performed analytically over $x$ and numerically over $\xi$. Numerical results are presented in Table I.

For two-loop vacuum polarization contributions into a potential (loop after loop term and 2-loop term) we have the following expressions which contain the same tensor operators $T_{i}$ as above [21, 23]:

$$
\begin{aligned}
& \Delta V_{1 \gamma, v p-v p}^{h f s}(r)=\frac{Z \alpha\left(1+\kappa_{d}\right)}{2 m_{1} m_{2} r^{3}}\left(\frac{\alpha}{3 \pi}\right)^{2} \int_{1}^{\infty} \rho(\xi) d \xi \int_{1}^{\infty} \rho(\eta) d \eta \frac{1}{\xi^{2}-\eta^{2}}\left[\left(1+\frac{m_{1} \kappa_{d}}{m_{2}\left(1+\kappa_{d}\right)}\right)\left(\boldsymbol{L} \cdot \boldsymbol{s}_{2}\right) \times\right. \\
& {\left[\xi^{2}\left(1+2 m_{e} \xi r\right) e^{-2 m_{e} \xi r}-\eta^{2}\left(1+2 m_{e} \eta r\right) e^{-2 m_{e} \eta r}\right]-\left(1+a_{\mu}\right)\left(4 m_{e}^{2} r^{2}\left[\xi^{4} e^{-2 m_{e} \xi r}-\eta^{4} e^{-2 m_{e} \eta r}\right] \times\right.} \\
& \left.\left.\left[\left(\boldsymbol{s}_{1} \cdot \boldsymbol{s}_{2}\right)-\left(\boldsymbol{s}_{1} \cdot \boldsymbol{n}\right)\left(\boldsymbol{s}_{2} \cdot \boldsymbol{n}\right)\right]+\left[\xi^{2}\left(1+2 m_{e} \xi r\right) e^{-2 m_{e} \xi r}-\eta^{2}\left(1+2 m_{e} \eta r\right) e^{-2 m_{e} \eta r}\right]\left[\left(\boldsymbol{s}_{1} \cdot \boldsymbol{s}_{2}\right)-3\left(\boldsymbol{s}_{1} \cdot \boldsymbol{n}\right)\left(\boldsymbol{s}_{2} \cdot \boldsymbol{n}\right)\right]\right)\right] \\
& \Delta V_{2-\text { loop vp }}^{\text {hfs }}(r)=\frac{Z \alpha\left(1+\kappa_{d}\right)}{2 m_{1} m_{2} r^{3}} \frac{2}{3}\left(\frac{\alpha}{\pi}\right)^{2} \int_{0}^{1} \frac{f(v) d v}{1-v^{2}} e^{-\frac{2 m_{e} r}{\sqrt{1-v^{2}}}}\left[\left(1+\frac{m_{1} \kappa_{d}}{m_{2}\left(1+\kappa_{d}\right)}\right)\left[1+\frac{2 m_{e} r}{\sqrt{1-v^{2}}}\right]\left(\boldsymbol{L} \cdot \boldsymbol{s}_{2}\right)-\right. \\
& \left.\left(1+a_{\mu}\right)\left(\frac{4 m_{e}^{2} r^{2}}{1-v^{2}}\left[\left(\boldsymbol{s}_{1} \cdot \boldsymbol{s}_{2}\right)-\left(\boldsymbol{s}_{1} \cdot \boldsymbol{n}\right)\left(\boldsymbol{s}_{2} \cdot \boldsymbol{n}\right)\right]+\left(1+\frac{2 m_{e} r}{\sqrt{1-v^{2}}}\right)\left[\left(\boldsymbol{s}_{1} \cdot \boldsymbol{s}_{2}\right)-3\left(\boldsymbol{s}_{1} \cdot \boldsymbol{n}\right)\left(\boldsymbol{s}_{2} \cdot \boldsymbol{n}\right)\right]\right)\right]
\end{aligned}
$$

After averaging (12) and (13) over wave functions we obtain numerical values of corresponding corrections to the HFS that are included in Table [. Numerically they are extremely small. Muonic VP correction of order $\alpha^{6}$ can de derived by means of simple replacement 
TABLE I: Numerical values of corrections to $2 P$-state hyperfine structure

\begin{tabular}{|c|c|c|c|c|c|c|c|}
\hline Contribution & $\begin{array}{c}2^{2} P_{1 / 2} \\
(\mu \mathrm{eV})\end{array}$ & $\begin{array}{c}2^{4} P_{1 / 2} \\
(\mu \mathrm{eV})\end{array}$ & $\begin{array}{c}2^{2} P_{3 / 2} \\
(\mu \mathrm{eV})\end{array}$ & $\begin{array}{c}2^{4} P_{3 / 2} \\
(\mu \mathrm{eV})\end{array}$ & $\begin{array}{c}2^{6} P_{3 / 2} \\
(\mu \mathrm{eV})\end{array}$ & $\begin{array}{c}2^{2} P_{1 / 2 \rightarrow 3 / 2} \\
(\mu \mathrm{eV})\end{array}$ & $\begin{array}{c}2^{4} P_{1 / 2 \rightarrow 3 / 2} \\
(\mu \mathrm{eV})\end{array}$ \\
\hline $\begin{array}{c}\text { Leading order } \\
\alpha^{4} \text { contribution }\end{array}$ & -1380.3359 & 690.1679 & 8162.2889 & 8583.2316 & 9284.8027 & -126.0372 & -199.2824 \\
\hline $\begin{array}{c}\text { Relativistic } \\
\text { correction of order } \alpha^{6}\end{array}$ & -0.1676 & 0.0838 & -0.0125 & -0.0050 & 0.0075 & -0.0043 & -0.0067 \\
\hline $\begin{array}{c}\text { VP corrections } \\
\text { of order } \alpha^{5}\end{array}$ & -1.0706 & 0.5353 & -0.2802 & -0.1121 & 0.1681 & -0.1437 & -0.2271 \\
\hline $\begin{array}{c}\text { VP corrections } \\
\text { of order } \alpha^{6}\end{array}$ & -0.0011 & 0.0005 & -0.0014 & -0.0006 & 0.0008 & 0.00005 & 0.0001 \\
\hline $\begin{array}{c}\text { Quadrupole } \\
\text { correction of order } \alpha^{4}\end{array}$ & 0 & 0 & 434.2329 & -347.3863 & 86.8466 & 614.0980 & -194.1948 \\
\hline $\begin{array}{c}\text { Quadrupole and VP } \\
\text { correction of order } \alpha^{5} \\
\text { in } 1 \gamma \text { interaction }\end{array}$ & 0 & 0 & 0.2438 & -0.1950 & 0.0488 & 0.3447 & -0.1090 \\
\hline $\begin{array}{c}\text { Quadrupole and VP } \\
\text { correction of order } \alpha^{5} \\
\text { in second order PT }\end{array}$ & 0 & 0 & 0.1122 & -0.0898 & 0.0224 & 0.1587 & -0.0502 \\
\hline Total value & -1381.5752 & 690.7876 & 8596.5838 & 8235.4428 & 9371.8969 & 488.4164 & -393.8702 \\
\hline
\end{tabular}

$m_{e}$ to $m_{1}$ in (4). One-loop VP contribution to the HFS in second order perturbation theory (SOPT) has the following general form [21, 23]:

$$
\Delta E_{S O P T \text { vp }}^{h f s}=2<\psi\left|\Delta V_{v p}^{C} \cdot \tilde{G} \cdot \Delta V_{B}^{h f s}\right| \psi>,
$$

where $\Delta V_{v p}^{C}(r)$ is the Coulomb potential modified by the vacuum polarization. The Coulomb Green's function $\tilde{G}$ for $2 P$-state was obtained in [26]. Substituting (11) and $\Delta V_{v p}^{C}(r)$ into (14) we get integral expression for the VP correction in SOPT:

$$
\begin{gathered}
\Delta E_{S O P T, v p}^{h f s}=\frac{\alpha^{5} \mu^{3}\left(1+\kappa_{d}\right)}{1296 \pi m_{1} m_{2}} \int_{1}^{\infty} \rho(\xi) d \xi \int_{0}^{\infty} d x e^{-x\left(1+\frac{2 m_{e} \xi}{W}\right)} \int_{0}^{\infty} \frac{e^{-x^{\prime}} d x^{\prime}}{x^{\prime 2}} g\left(x, x^{\prime}\right) \times \\
{\left[\left(1+\frac{m_{1} \kappa_{d}}{m_{2}\left(1+\kappa_{d}\right)}\right) \overline{T_{1}}-\left(1+a_{\mu}\right) \overline{T_{2}}\right] .}
\end{gathered}
$$

The integration in (15) is performed analytically over $x, x^{\prime}$ and numerically over $\xi$. For twoloop contributions in second order PT we use the potential (3) and the VP modifications of the Coulomb potential from [21, 23]. Corresponding numerical results are included in Table I. The contribution of the VP of order $\alpha^{6}$ to the HFS of P-states in third order PT is estimated using the known formulas. Omitting here intermediate analytical expressions (see [7]) we have included numerical value in Table [1. 
The deuteron has a non-zero quadrupole moment which leads to additional quadrupole interaction correction of order $\alpha^{4}$ to hyperfine structure of P-states [17]. We turn right to the calculation of the quadrupole corrections for the effects of vacuum polarization. To construct the required interaction operator we use the multipole expansion of the Coulomb potential, taking into account the effect of vacuum polarization:

$$
\begin{gathered}
V_{v p}^{C}(r)=-Z e^{2} \frac{\alpha}{3 \pi} \int_{1}^{\infty} \rho(\xi) d \xi \int \frac{\rho\left(r^{\prime}\right) d \mathbf{r}^{\prime}}{\left|\mathbf{r}-\mathbf{r}^{\prime}\right|} e^{-2 m_{e} \xi\left|\mathbf{r}-\mathbf{r}^{\prime}\right|}= \\
-Z e^{2} \frac{\alpha}{3 \pi r} \int_{1}^{\infty} \rho(\xi) e^{-2 m_{e} \xi r} d \xi \int \rho\left(r^{\prime}\right) d \mathbf{r}^{\prime}\left[P_{0} f_{0}\left(r, r^{\prime}, \xi\right)+P_{1} f_{1}\left(r, r^{\prime}, \xi\right)+P_{2} f_{2}\left(r, r^{\prime}, \xi\right)+\ldots\right],
\end{gathered}
$$

where $P_{n}(\cos \theta)$ are the Legendre polynomials, $\rho\left(r^{\prime}\right)$ is the nuclear density,

$f_{0}\left(r, r^{\prime}, \xi\right)=1+\frac{r^{\prime 2}}{r^{2}} \frac{4 m_{e}^{2} \xi^{2} r^{2}}{6}, f_{1}\left(r, r^{\prime}, \xi\right)=\frac{r^{\prime}}{r}\left(1+2 m_{e} \xi r\right), f_{2}\left(r, r^{\prime}, \xi\right)=\frac{r^{\prime 2}}{r^{2}}\left(1+2 m_{e} \xi r+\frac{4 m_{e}^{2} \xi^{2} r^{2}}{3}\right)$.

A separation of the muon and nuclear variables in (16) can be made using the addition theorem for spherical harmonics. As a result, the expression (16) is converted into a sum of scalar products of irreducible tensors. The third term in square brackets in (16) is determined by the nuclear quadrupole momentum. Then the energy of quadrupole interaction with vacuum polarization correction can be presented in the form:

$$
\begin{gathered}
\Delta E_{v p}^{Q}=-Z e^{2}<F M I j^{\prime}\left|Q_{20}(d) \cdot T_{20}^{v p}(\mu)\right| F M I j>= \\
-Z e^{2}(-1)^{I+j^{\prime}-F} W\left(j I j^{\prime} I ; F 2\right)<j^{\prime}|| T_{20}^{v p}(\mu)\|j><I\| Q_{20}(d) \| I>,
\end{gathered}
$$

where nuclear quadrupole tensor and a tensor of muon cloud of rank 2 are equal correspondingly

$$
\begin{gathered}
Q_{20}(d)=\sqrt{\frac{4 \pi}{5}} r^{\prime 2} Y_{20}\left(\theta^{\prime}, \phi^{\prime}\right)=\frac{3 z^{\prime 2}-r^{\prime 2}}{2}, \\
T_{20}^{v p}(\mu)=\frac{\alpha}{3 \pi} \int_{1}^{\infty} \rho(\xi) d \xi \frac{e^{-2 m_{e} \xi r}}{r^{3}}\left(1+2 m_{e} \xi r+\frac{4 m_{e}^{2} \xi^{2} r^{2}}{3}\right) \sqrt{\frac{4 \pi}{5}} Y_{20}(\theta, \phi) .
\end{gathered}
$$

The nuclear quadrupole momentum $Q$ is determined by reduced matrix element of deuteron tensor $Q_{20}(d)[24]$ :

$$
<I|| Q_{20}(d) \| I>=\frac{Q}{2}\left[\left(\begin{array}{ccc}
I & 2 & I \\
-I & 0 & I
\end{array}\right)\right]^{-1},
$$

In turn, the reduced matrix element of muon tensor differs from the case of the quadrupole correction of the leading order only by the value of radial integral:

$$
<j^{\prime}|| T_{20}^{v p}(\mu) \| j>=\sqrt{2 j+1} \sqrt{2 j^{\prime}+1}(-1)^{j^{\prime}+1 / 2}\left(\begin{array}{ccc}
j^{\prime} & 2 & j \\
\frac{1}{2} & 0 & -\frac{1}{2}
\end{array}\right)<\frac{e^{-2 m_{e} \xi r}}{r^{3}}\left(1+2 m_{e} \xi r+\frac{4 m_{e}^{2} \xi^{2} r^{2}}{3}\right)>,
$$

Substituting (21) and (22) into (18) we obtain the quadrupole interaction contribution with the account of vacuum polarization effects in calculating both the diagonal and offdiagonal matrix elements in the form:

$$
\Delta E_{v p}^{Q}=(-1)^{j^{\prime}+1 / 2-F-j}\left\{\begin{array}{lll}
j & I & F \\
I & j^{\prime} & 2
\end{array}\right\} \frac{Q}{2}\left[\left(\begin{array}{ccc}
I & 2 & I \\
-I & 0 & I
\end{array}\right)\right]^{-1} \times
$$




$$
\times \sqrt{2 j+1} \sqrt{2 j^{\prime}+1}\left(\begin{array}{ccc}
j^{\prime} & 2 & j \\
\frac{1}{2} & 0 & -\frac{1}{2}
\end{array}\right)<\frac{Z \alpha^{2} e^{-2 m_{e} \xi r}}{r^{3}}\left(1+2 m_{e} \xi r+\frac{4 m_{e}^{2} \xi^{2} r^{2}}{3}\right)>.
$$

For diagonal and off-diagonal matrix elements we get following analytical integral expressions with subsequent numerical evaluation:

$$
\begin{aligned}
\Delta E_{v p}^{Q}\left(j^{\prime}=3 / 2, j=3 / 2\right) & =\frac{\mu^{3} \alpha(Z \alpha)^{4} Q}{36 \pi} \int_{1}^{\infty} \frac{\left(5 a^{2}+8 a+4\right)}{(a+2)^{4}} \rho(\xi) d \xi\left[\delta_{F \frac{1}{2}}-\frac{4}{5} \delta_{F \frac{3}{2}}+\frac{1}{5} \delta_{F \frac{5}{2}}\right]= \\
& =\left[\delta_{F \frac{1}{2}}-\frac{4}{5} \delta_{F \frac{3}{2}}+\frac{1}{5} \delta_{F \frac{5}{2}}\right] \times 0.2441 \mu e V, \\
\Delta E_{v p}^{Q}\left(j^{\prime}=1 / 2, j=3 / 2\right) & =\frac{\mu^{3} \alpha(Z \alpha)^{4} Q}{36 \pi} \int_{1}^{\infty} \frac{\left(5 a^{2}+8 a+4\right)}{(a+2)^{4}} \rho(\xi) d \xi\left[\sqrt{2} \delta_{F \frac{1}{2}}-\frac{1}{\sqrt{5}} \delta_{F \frac{3}{2}}\right]= \\
& =\left[\sqrt{2} \delta_{F \frac{1}{2}}-\frac{1}{\sqrt{5}} \delta_{F \frac{3}{2}}\right] \times 0.2441 \mu e V,
\end{aligned}
$$

where the value of deuteron quadrupole moment is equal to $Q=0.285783(30) \mathrm{fm}^{2}$ [27], $a=4 m_{e} \xi / \mu \alpha$. Note that numerical coefficients in (24) and (25) coincide. The quadrupole and VP corrections to diagonal and off-diagonal matrix elements in second order PT which can be calculated as in (14) are equal to

$$
\begin{gathered}
E_{\text {sopt }, v p}^{Q}\left(j^{\prime}=3 / 2, j=3 / 2\right)=\left[\delta_{F \frac{1}{2}}-\frac{4}{5} \delta_{F \frac{3}{2}}+\frac{1}{5} \delta_{F \frac{5}{2}}\right] \times 0.1122 \mu \mathrm{eV}, \\
E_{\text {sopt }, v p}^{Q}\left(j^{\prime}=1 / 2, j=3 / 2\right)=\left[\sqrt{2} \delta_{F \frac{1}{2}}-\frac{1}{\sqrt{5}} \delta_{F \frac{3}{2}}\right] \times 0.1122 \mu \mathrm{eV} .
\end{gathered}
$$

In this work, we have continued to study the P-states HFS in muonic deuterium, which began in [17]. If in [17] a method for HFS calculating of P-levels in momentum representation was formulated, in this paper we have used the formalism of irreducible tensor operators in the calculation of corrections in the coordinate representation. Both representations are complementary to each other, so that the calculation of the matrix elements of the operators in both representations is in our opinion useful. The results obtained here and in [17] are in agreement, but we are able to correct technical inaccuracy in the calculation of the off-diagonal matrix elements in [17] (see (25)). We present corrections in integral form and evaluate them numerically. After diagonalization of the results from the energy matrix in Table 1 we obtain final values of $2 P$-state hyperfine structure in muonic deuterium: $E_{1 / 2}^{F=1 / 2}=-1405.4254 \mu \mathrm{eV}, E_{1 / 2}^{F=3 / 2}=670.2812 \mu \mathrm{eV}, E_{3 / 2}^{F=1 / 2}=8620.4340 \mu \mathrm{eV}, E_{3 / 2}^{F=3 / 2}=$ $8255.9492 \mu \mathrm{eV}, E_{3 / 2}^{F=5 / 2}=9371.8969 \mu \mathrm{eV}$. The detailed calculation of 2P-state HFS in muonic deuterium was performed in [4], where first order PT vacuum polarization corrections were included. Vacuum polarization corrections in [4] were evaluated approximately thus they differ from our values (4) by $\sim 30 \%$. Other differences are connected with second order PT $\alpha^{5}$ and $\alpha^{6}$ corrections. Obtained results can be used for improved estimates of transition frequencies between $2 P$ and $2 S$ states regarding to the CREMA experiments.

We are grateful to F. Kottmann, R. Pohl and B. Franke for useful discussions and information about recent experimental results of the CREMA collaboration. The work is 
supported by the Russian Foundation for Basic Research (grant 16-02-00554) and the Ministry of Education and Science of Russia under grant No. 1394.

[1] A. Antognini et al., Nature. 466, 213 (2010).

[2] A. Antognini et al., Science. 339, 417 (2013).

[3] R Pohl, F. Nez, T. Udem et al. arXiv:1607.03165[physics.atom-ph].

[4] E. Borie, Ann. Phys. 327, 733 (2012).

[5] E. Borie, Z. Phys. A 278, 127 (1976).

[6] M. I. Eides, H. Grotch, V. A. Shelyuto, Phys. Rep. 342, 63 (2001).

[7] R. N. Faustov, A. P. Martynenko, G. A. Martynenko, V. V. Sorokin, Phys. Rev. A 90, 012520 (2014).

[8] R. N. Faustov, A. P. Martynenko, G. A. Martynenko, V. V. Sorokin, Phys. Lett. B 733, 354 (2014).

[9] R. Pohl, R. Gilman, G. A. Miller, and K. Pachucki, Annu. Rev. Nucl. Part. Sci. 63, 175 (2013).

[10] J. J. Krauth, M. Diepold, B. Franke et al. Ann. Phys. 366, 168 (2016).

[11] G. A. Miller, A. W. Thomas, J. D. Carroll, and J. Rafelski, Phys. Rev. A 84, 020101(R) (2011).

[12] S. G. Karshenboim, E. Yu. Korzinin, V. G. Ivanov and V. A. Shelyuto, Phys. Rev. A 81, 060501 (2010).

[13] U. D. Jentschura, Ann. Phys. 326, 500 (2011).

[14] C. E. Carlson, V. Nazaryan and K. Griffioen, Phys. Rev. A 83, 042509 (2011).

[15] P. Indelicato, Phys. Rev. A 87, 022501 (2013).

[16] C. Peset and A. Pineda, Eur. Phys. J. A 51, 156 (2015).

[17] R. N. Faustov, A. P. Martynenko, G. A. Martynenko and V. V. Sorokin, Phys. Rev. A 92, $052512(2015)$.

[18] R. N. Faustov and A. P. Martynenko, J. Exp. Theor. Phys. 88, 672 (1999); J. Exp. Theor. Phys. 98, 39 (2004).

[19] A. P. Martynenko, J. Exp. Theor. Phys. 101, 1021 (2005).

[20] A. A. Krutov, A. P. Martynenko, G. A. Martynenko, R. N. Faustov, J. Exp. Theor. Phys. 120, 73 (2015).

[21] E. N. Elekina and A. P. Martynenko, Phys. Atom. Nucl. 73, 1828 (2010).

[22] S. J. Brodsky and R. G. Parsons, Phys. Rev. 163, 134 (1967).

[23] A. P. Martynenko, Phys. Atom. Nucl. 71, 125 (2008).

[24] I. I. Sobelmann, Introduction to Theory of Atomic Spectra (Pergamon Press, London, 1968).

[25] G. T. Emery, Hyperfine structure, In Handbook of Atomic, Molecular, and Optic Physics, Gordon W. F. Drake (Ed.), NY, Springer. 2006.

[26] H. F. Hameka, J. Chem. Phys. 47, 2728 (1967).

[27] M. Pavanello, W.-C. Tung, L. Adamowicz, Phys. Rev. A 81, 042526 (2010). 